\title{
THE SINGULARITIES OF THE MODULUS SPACE ${ }^{1}$
}

\author{
BY H. E. RAUCH
}

Communicated by R. Bott, March 21, 1962

The results collected in the following theorem are contained explicitly in the literature $([1 ; 2 ; 8 ; 3]$ and references there) or are known to workers in the field and readily deduced from the literature.

TheOREM A. (i) the set of conformal equivalence classes of compact Riemann surfaces (henceforth: classes) of fixed genus $g \geqq 2$ can be endowed with the structure of a normal analytic space $M^{g}$ of complex $d i$ mension $3 g-3$. This structure is derived from the structure of a complex analytic manifold, the Torelli space $\mathfrak{I}^{\sigma}$, by identification under the action of a properly discontinuous group of analytic automorphisms, the reduced mapping class (Siegel modular) group $G^{g}$. In this description $\mathfrak{T}^{g}$ appears as a branched covering of $M^{g} . P \in \Delta^{g}$, the branch locus, if and only if it is a fixed point of a nontrivial finite subgroup $G^{g}(P)$ of $G^{g}$, in which case $P$ lies over a class (an $H$-class) in $M^{o}$ containing a surface $S$ (H-surface) admitting at least one nontrivial conformal automorphism and $G^{g}(P)$ is isomorphic with $H(S)$, the automorphism group of $S$.

(ii) The (faithful) representation of $G^{g}(P)$ at $P \in \Delta^{g}$ by its differential action is concretely represented by linear transformations (nonsingular) on the space of analytic (finite) quadratic differentials on any $S$ in the class under $P$.

The question arises: which points of $M^{g}$ are uniformizable, i.e., manifold points? Clearly, by Theorem A, nonuniformizable pointshenceforth called singular points or singularities-occur at most among the points under $\Delta^{g}$. The purpose of this note is to establish

THEOREM 1. (i) The only singularity under $\Delta^{2}$ is the class containing the surface with the equation $y^{2}=x^{6}-x$.

(ii) Under $\Delta^{3}$ the $H$-classes containing hyperelliptic surfaces with no other automorphisms are uniformizable; all other points under $\Delta^{3}$ are singular.

(iii) Every point under $\Delta^{o}, g \geqq 4$, is singular.

Part (i) of Theorem 1 is due to Gerstenhaber (unpublished), Fischer [4], and Igusa [6] and is easily established by the methods of this note with the aid of some computation. In [4] it is established that the hyperelliptic classes of genus 3 with additional automorphisms are singularities of the hyperelliptic modulus space-a result

${ }^{1}$ Partially supported by NSF Grant \#G18929. 
also easily established by the present methods-but that does not imply (ii).

One needs three basic tools:

Criterion B. $A$ n.a.s.c. for $\bar{P} \in M^{g}$ under $P \in \Delta^{g}$ to be uniformizable is that the ring $\Omega(P)$ of germs of holomorphic functions at $P$ vanishing there which are invariant under $G^{g}(P)$ contain $3 g-3$ elements generating all of $\Omega(P)$ as power series.

CRITERION C (purity of the branch locus). If $P \in \Delta^{o}$, a necessary condition for the point $\bar{P} \in M^{g}$ lying under $P$ to be uniformizable is that $\Delta^{g}$ be pure $3 g-4$ dimensional near $P$.

Theorem D. Let $P \in \Delta^{g}$, let $S$ belong to the class under $P$, and let $H_{0} \subset H(S)$ be a nontrivial subgroup and let $G_{0}(P) \subset G(P)$ be the corresponding subgroup under the isomorphism of Theorem A. Then near $P$ the set of fixed points in $\mathfrak{I}^{g}$ of $G_{0}(P)$ is a complex analytic submanifold of $\Delta^{g}$ whose tangent space at $P$ is spanned by the set of quadratic differentials on $S$ which are invariant under $H_{0}$. In particular, to determine the branches of $\Delta^{o}$ through $P$ it is sufficient to determine the sets of maximum dimension of quadratic differentials on $S$ invariant under nontrivial subgroups of $H(S)$.

Criterion $\mathrm{B}$ is a consequence of the construction of $M^{g}$ indicated in Theorem A [3] and the definition of uniformizable point. It is reminiscent of Zariski's criterion for a simple point of an algebraic variety [10]. The only reference of which $\mathrm{I}$ am aware at this writing for Criterion $\mathrm{C}$ is [11] where the theorem is proved for algebraic varieties over abstract fields. It should, however, in the present case - algebroid over the complex field-be a straightforward application of the algebra and function theory of several complex variables. Theorem $\mathrm{D}$ is proved by an extension of the method $[9 ; 8]$ I used to analyze the structure of the hyperelliptic sublocus of $\mathfrak{T}^{g}$ and is developed in Lewittes' Yeshiva Thesis.

To establish (ii) one observes that if $S$ is hyperelliptic it can be represented by the equation $y^{2}=P(x), P(x)$ of degree eight. One computes the quadratic differentials explicitly as

$$
\zeta_{1}=\frac{d x^{2}}{y^{2}}, \ldots, \zeta_{5}=\frac{x^{4} d x^{2}}{y^{2}}, \quad \zeta_{6}=\frac{d x^{2}}{y} .
$$

The automorphism $J: y \rightarrow-y, x \rightarrow x$ is represented by $(1, \cdots, 1,-1)$ and one sees that if there is no other automorphism the ring $\Omega(P)$ of Criterion B over the class of $S$ is isomorphic in a natural manner, by Theorem $A$, to the ring of germs of power series in $\zeta_{1}, \cdots, \zeta_{5}, \zeta_{6}^{2}$ which are $6=3 \cdot 3-3$ in number and Criterion B 
applies. If $S$ admits another automorphism $H$ of period $n$, then [5, p. 256] one can choose as equation $y^{2}=Q\left(x^{n}\right)$ or $y^{2}=x R\left(x^{n}\right)$ where $Q$ and $R$ are polynomials of appropriate degrees. Thus the only possibilities are $H_{2}: x \rightarrow-x, n=2 ; H_{4}: x \rightarrow i x, n=4 ; H_{7}: x \rightarrow \epsilon x, y \rightarrow \epsilon^{1 / 2} y$, $\epsilon^{7}=1, \epsilon \neq 1, n=7$. One convinces oneself that the cases $n=2,4$ or $n=7$ are mutually exclusive. Now $\zeta_{1}, \zeta_{3}, \zeta_{5}, \zeta_{6}$ are invariant under $H_{2}$ and $\zeta_{1}, \cdots, \zeta_{5}$ under $J$ so that $\Delta^{3}$ over the class of $S$ by Theorem $\mathrm{D}$ consists of two branches of dimension 4 and 5 respectively, and by Criterion $\mathrm{C}$ the class is singular. If $S$ admits $H_{4}$ it admits $H_{2}$ and its class is the limit of classes admitting $\mathrm{H}_{2}$ and so is singular. If $S$ admits $H_{7}$ then $\Omega(P)$ is isomorphic to the power series in the minimal set containing $\zeta_{1}^{7}, \cdots, \zeta_{5}^{7}, \zeta_{6}^{14}, \zeta_{1} \zeta_{2}^{6}$ among others so that more than $3 g-3=6$ elements are needed and Criterion B shows that the point is singular.

Now for $g \geqq 4$ in the hyperelliptic case the involution $J$ again has representation $(1, \cdots, 1,-1, \cdots,-1)$ on a suitable basis, the last bloc having $g-2 \geqq 2$ members, whence a similar reasoning leads to the singular character provided no other automorphisms are present. But the points with more automorphisms are limits of those without.

To deal with the nonhyperelliptic surfaces for $g \geqq 3$ one needs the important result of Lewittes [7]:

Theorem E. An automorphism $H$ of prime order $N$ on $S, g \geqq 3$, has invariant subspace of quadratic differentials of dimension at most $3 g-5$ if $S /\{H\}$ has genus $g_{1} \geqq 1$, where $\{H\}$ is the cyclic subgroup of $H(S)$ generated by $H$.

At the end of this note I prove the following supplement:

THEOREM $\mathrm{E}_{1}$. The conclusion of Theorem $\mathrm{E}$ holds when $g_{1}=0$ and $S$ is nonhyperelliptic.

To complete the proof of Theorem 1 one sees that Theorems $\mathrm{E}$ and $E_{1}$ imply by Theorem $D$ that Criterion $C$ is violated for all nonhyperelliptic classes with $g \geqq 3$ since every nontrivial subgroup of any $H(S)$ contains a cyclic subgroup of prime order and the dimension of the invariant subspace is a fortiori at most $3 g-5$.

To prove Theorem $E_{1}$, observe that, if $g_{1}=0$, by [5, p. 246] one can represent $S$ by $y^{N}=P_{m}(x)=\left(x-\alpha_{1}\right)^{n_{1}} \cdots\left(x-\alpha_{l}\right)^{n_{l}}$, where $N \nmid m=\sum m_{i}, 1 \leqq m_{i} \leqq N-1 . \alpha_{1}, \cdots, \alpha_{l}, \infty$, are under the $l+1$ fixed points of $H: y \rightarrow \epsilon y, \epsilon^{N}=1, \epsilon \neq 1$. The claim is that for $g \geqq 3$, $N \geqq 3$ ( $N=2$ is hyperelliptic case) 


$$
\zeta_{1}=\frac{\prod\left(x-\alpha_{i}\right)^{2 m_{i}-2} d x^{2}}{y^{2 N-2}}, \quad \zeta_{2}=\frac{(x-\alpha) \prod\left(x-\alpha_{i}\right)^{2 m_{i}-2} d x^{2}}{y^{2 N-2}},
$$

where $\alpha \neq \alpha_{i}, i=1, \cdots, l$, are noninvariant finite quadratic differentials. The noninvariance is obvious, $N \geqq 3$ being prime. Now the points over $x=\alpha_{1}, \cdots, \alpha_{l}, \infty$ are $(N-1)$ st order branch points, and one finds that $\zeta_{1}, \zeta_{2}$ are finite at the finite ones since $\left(x-\alpha_{i}\right)=t^{N}$, $y \sim t^{m_{i}}$ for $x$ near $\alpha_{i}$ and

$$
\zeta_{1}, \zeta_{2} \sim t^{2 N\left(m_{i}-1\right)+2(N-1)-2(N-1) m_{i}} d t^{2}=t^{2\left(m_{i}-1\right)} d t^{2} .
$$

Near $x=\infty, x=1 / t^{N}, y \sim 1 / t^{m}, \zeta_{2} \sim t^{-N-2(m-l)-2(N+1)+2(N-1) m} d t^{2}$ $=t^{2(N-2) m+2 l}-{ }^{3 N-2} d t^{2}$, which is finite if

$$
m \geqq \frac{3 N-2(l-1)}{2 N-4}=\frac{3}{2}+\frac{4-l}{N-2} \text {. }
$$

But the last expression is at most $3 / 2+2=7 / 2$ since $N \geqq 3$ and $l \geqq 2$ (the number of fixed points for $g_{1}=0$ is at least $3[5$, p. 245]). Thus for $m \geqq 4$ the proof is finished. If $m=2$, then $l=2$ and $g=(l-1)(N-1) / 2$ $=(N-1) / 2 \geqq 3$ only for $N \geqq 7$ in which case $m=2>1.9=3 / 2+2 / 5$ $\geqq 3 / 2+(4-2) /(N-2)$. If $m=3, l=2, g=(N-1) / 2 \geqq 3$ for $N \geqq 7$, and the preceding applies, or $l=3, g=(N-1) \geqq 3$ for $N \geqq 5$ (prime), in which case $m=3>11 / 6=3 / 2+1 / 3 \geqq 3 / 2+(4-l) /(N-2)$. When $\zeta_{2}$ is finite $\zeta_{1}$ is, too.

While discussing the contents of this note with me, Zariski and Mumford pointed out that a quick heuristic count of the moduli of curves admitting a given prime order automorphism gives $3 g_{1}-3+t$, this being the number of moduli of the collapsed curve plus the number of (movable) branch points; whence a comparison of this number with $3 g-3=3 / 2(N-1) t+N\left(3 g_{1}-3\right)$ deduced from Riemann-Hurwitz gives directly that the codimension of $\Delta^{g}$ is at least two for $g_{1} \geqq 2$ and then, with some maneuvering, for the remaining cases. I pointed out that $\left(3 g_{1}-3\right)+t$ is rigorously established by (i) of Lewittes' Theorem 1 whereupon the remainder of their argument corresponds to an alternative derivation of Theorem $\mathrm{E}$. They noted that Mumford's work on the modulus variety gives a correct algebraic justification of the heuristic count.

\section{REFERENCES}

1. L. Ahlfors, The complex analytic structure of the space of closed Riemann surfaces, Analytic functions, pp. 45-60, Princeton Univ. Press, Princeton, N. J., 1960.

2. L. Bers, Spaces of Riemann surfaces, Proc. Int. Cong. Math. Edinburgh, 1958, pp. 349-361. 
3. H. Cartan, Quotient d'un espace analytique par un groupe d'automorphismes, Algebraic geometry and topology, Princeton, 1957, pp. 90-102.

4. I. Fischer, The moduli of hyperelliptic curves, Trans. Amer. Math. Soc. 82 (1956), 64-84.

5. A. Hurwitz, Mathematische Werke, Bd. 1, Basel, 1932.

6. J. Igusa, Arithmetic variety of moduli for genus two, Ann. of Math. (2) 72 (1960), $612-649$.

7. J. Lewittes, Invariant quadratic differentials, Bull. Amer. Math. Soc. 68 (1962), $320-322$.

8. H. E. Rauch, Variational methods in the problem of the moduli of Riemann surfaces, Contributions to function theory, Bombay, 1960, pp. 17-40.

9. - On the moduli of Riemann surfaces, Proc. Nat. Acad. Sci. U.S.A. 41 (1955), 236-238.

10. O. Zariski, The concept of a simple point of an abstract algebraic variety, Trans. Amer. Math. Soc. 62 (1947), 1-52.

11. - The purity of the branch locus of algebraic functions, Proc. Nat. Acad. Sci. U.S.A. 44 (1958), 791-796.

YeshIVA UNIVERSITY 